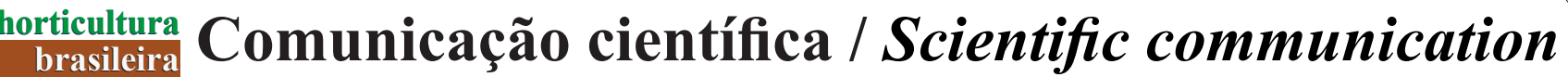

ANDRADE JÚNIOR VC; GOMES JAA; OLIVEIRA CM; AZEVEDO AM; FERNANDES JSC; GOMES, LAA; MALUF WR. 2016. Resistência de clones de batata-doce a Meloidogyne javanica. Horticultura Brasileira 34: 130-136. DOI - http://dx.doi.org/10.1590/S0102-053620160000100020

\section{Resistência de clones de batata-doce a Meloidogyne javanica}

\author{
Valter C Andrade Júnior; Jorge AA Gomes'; Celso M Oliveira²; Alcinei M Azevedo ; José SC Fernandes ${ }^{1}$; \\ Luiz AA Gomes' ${ }^{2}$ Wilson R Maluf ${ }^{2}$ \\ ${ }^{1}$ Universidade Federal dos Vales do Jequitinhonha e Mucuri (UFVJM), Diamantina-MG; Brasil; valterjr15@gmail.com; jorge. \\ agronomia@yahoo.com.br; cunha.fernandes@yahoo.com.br; ${ }^{2}$ Universidade Federal de Lavras (UFLA), Lavras-MG, Brasil; \\ celsodoliveira@yahoo.com.br, laagomes@dag.ufla.br; ${ }^{3}$ Universidade Federal de Viçosa (UFV), Viçosa-MG, Brasil; alcineimistico@ \\ hotmail.com
}

\section{RESUMO}

O objetivo deste trabalho foi avaliar e selecionar clones de batata-doce (Ipomoea batatas) quanto à resistência à Meloidogyne javanica. Avaliaram-se 63 clones, dentre eles, cultivares comerciais (Brazlândia Rosada, Brazlândia Branca, Palmas, Princesa e Coquinho) além do tomateiro cv. Santa Clara (suscetível ao Meloidogyne spp.). As ramas foram plantadas em badejas de poliestireno expandido de 72 células com substrato comercial e mantidas em casa de vegetação. $\mathrm{O}$ delineamento experimental utilizado foi de blocos casualizados com três repetições e seis plantas por parcela. A inoculação do patógeno foi feita 30 dias após o plantio das ramas e, após 75 dias, as ramas foram retiradas das bandejas e suas raízes lavadas. Foi realizada a extração dos ovos dos nematoides e procedeu-se a sua contagem. A classificação dos níveis de resistência foi realizada pelo fator de reprodução (FR) e o índice de reprodução (IR). Dos 63 clones avaliados $71,43 \%$ foram identificados como resistentes pelo fator de reprodução e $82,51 \%$ foram classificados como altamente resistente ou muito resistente pelo índice de reprodução. Pelas duas classificações de resistência utilizadas, foram selecionados, 71,43\% clones de batata-doce resistentes a Meloidogyne javanica.

Palavras-chave: Ipomoea batatas, Meloidogyne spp., nematoidesdas-galhas, fator de reprodução, índice de reprodução.

\begin{abstract}
Resistance of sweet potato clones to Meloidogyne javanica

The objective of this study was to evaluate and select clones of sweet potato (Ipomoea batatas) for their resistance to Meloidogyne javanica. Sixty-tree clones were evaluated, among the commercial cultivars (Brazlândia Rosada, Brazlândia Branca, Palmas, Princesa and Coquinho) plus the tomato cv. Santa Clara (susceptible to Meloidogyne spp.). The stems were planted in expanded polystyrene trays with 72 cells with commercial substrate and maintained in a greenhouse. The experimental setup was a randomized blocks design with three replications and six plants per plot. The pathogen inoculation was carried out 30 days after the stems planting, and after 75 days the stems were removed from the polystyrene trays and their roots were washed. Extraction and counting of nematode eggs were carried out. Classifying resistance levels was carried out based on reproductive factors (FR) and reproduction index (IR). Of the 63 clones $71.43 \%$ were identified as resistant by the reproduction factor and $82.51 \%$ were classified as highly resistant or very resistant by reproduction index. When the both classification criteria were used, $71.43 \%$ of sweet potato clones were classified as resistant to Meloidogyne javanica.
\end{abstract}

Keywords: Ipomoea batatas, Meloidogyne spp., root-knot nematodes, reproduction factor, reproduction index.

(Recebido para publicação em 16 de setembro de 2014; aceito em 31 de julho de 2015) (Received on September 16, 2014; accepted on July 31, 2015)

$\mathrm{N}$ o mundo, a batata-doce (Ipomoea batatas) é a sexta cultura alimentar mais importante depois do arroz, trigo, batata, milho e mandioca. A Ásia é a maior região produtora de batata-doce do mundo com aproximadamente $80 \%$ da produção (CIP, 2013). O Brasil é o principal produtor no continente latino americano, obtendo em 2011, produtividade média de 12,4 t/ha em uma área plantada de 43.879 ha. Minas Gerais se destaca com a produção de $46.653 \mathrm{t} \mathrm{em}$ 2.672 ha de área cultivada (IBGE, 2011).

É considerada uma cultura rústica, devido a sua ampla adaptabilidade, tolerância à seca, pouca exigência em fertilidade além de ser bem produtiva em solos pobres e degradados (Silva et al., 2008). Embora apresente esses fatores é suscetível a um grande número de doenças causadas por fitopatógenos bem como ao ataque de inúmeras pragas que podem limitar sua produtividade. Dentre os fitopatógenos destacam-se os nematoides do gênero Meloidogyne, também conhecidos como nematoides-das-galhas. Em razão da sua distribuição mundial, ampla gama de hospedeiros e capacidade de interação com outras espécies fitopatogênicas, este grupo de fitonematóides tounou-se um dos maiores causadores de prejuízos na agricultura (Perry et al., 2009), e consequentemente, para o agricultor (Neves et al., 2010). De acordo com Wesemael et al. (2011), embora Meloidogyne seja considerado em todo o mundo como o gênero mais importante de fitonematoides, informações na literatura científica sobre o impacto econômico dos nematoides-das-galhas são escassos.

Várias espécies de Meloidogyne 
têm sido encontradas em campos de produção, incluindo $M$. arenaria, $M$. hapla, M. enterolobii, M. incognita e M. javanica (Silva et al., 2011). Meloidogyne javanica é o segundo nematoide das galhas em ordem de importância que causa perda qualitativa à cultura da batata-doce, depois de Meloidogyne incognita (Jatala, 1991). No Brasil, as espécies mais importantes no cultivo de batata-doce são Meloidogyne. incognita raças 1, 2, 3 e 4 e Meloidogyne javanica (Charchar \& Ritschel, 2004).

Os sintomas da meloidoginose podem ser os mais diversos, incluindo necrose radicular, galhas radiculares, deficiências nutricionais, ramificação anormal das raízes, murcha e clorose. $\mathrm{O}$ processo de infecção ocorrendo no sistema radicular contribui para entrada de outros fitopatógenos além de predispor a planta a estresses ambientais, o que prejudica seu desenvolvimento (Marino et al., 2012). No entanto, a formação de galhas com tamanho e formato variados, é o sintoma mais comum. Segundo Charchar \& Ritschel (2004), a batata-doce é considerada como "falsa hospedeira", devido suas raízes secundárias apresentarem grande potencial para acumular alta população dos nematoides com a presença de inúmeras fêmeas que produzem massas de ovos imperceptíveis a olho nu, sem formação de galhas.

Várias medidas de manejo de Meloidogyne spp. são recomendadas, a exemplo da fumigação do solo, da seleção de material de propagação sem a presença de nematoide, da rotação com culturas não hospedeiras, do controle biológico, do uso de produtos naturais e de cultivares resistentes (Echeverrigaray et al., 2010). Devido aos problemas ambientais causados pelos nematicidas, além de geralmente ser uma prática antieconômica, requerer mão-de-obra na aplicação, apresentar alto custo e nem sempre ser eficiente (Pinheiro et al., 2009), a utilização de cultivares resistentes é de grande importância econômica e provavelmente o método ambientalmente mais seguro (Wesemael et al., 2011).

De acordo com Fiorini et al. (2007), a busca por fontes de resistência aos nematoides, bem como a procura pelo desenvolvimento de cultivares resistentes, adaptadas às diversas condições brasileiras, tem sido preocupação de pesquisadores brasileiros, especialmente a partir do início da década de 1990. Assim, o objetivo deste trabalho foi selecionar e avaliar a resistência de clones de batata-doce ao nematoide, Meloidogyne javanica.

\section{MATERIAL E MÉTODOS}

O experimento foi conduzido em casa de vegetação na Estação Experimental de Hortaliças da HortiAgro Sementes Ltda., Fazenda Palmital, Município de Ijaci-MG e as avaliações foram realizadas no Laboratório de Nematologia do Departamento de Fitopatologia, da Universidade Federal de Lavras, em Lavras-MG, no período de janeiro a abril de 2013.

Foram avaliados 63 clones de batata-doce, sendo 58 provenientes do banco de germoplasma da Universidade Federal dos Vales do Jequitinhonha e Mucuri, além de cinco cultivares comerciais de batata-doce (Brazlândia Rosada, Brazlândia Branca, Palmas, Princesa e Coquinho) e da cultivar de tomate Santa Clara (Solanum lycopersicum), utilizada como padrão de suscetibilidade ao Melodogyne spp. e controle. O banco de germoplasma da UFVJM sofreu no ano de 2013 uma reforma de padronização nominal, sendo os códigos antigos e atuais apresentados na tabela 1 .

Utilizou-se o delineamento experimental de blocos ao acaso com três repetições e seis plantas por parcela. Cada bloco foi composto por seis bandejas de poliestireno expandido de 72 células, preenchidas com aproximadamente 120 $\mathrm{mL}$ de substrato comercial Plantmax. Cada bandeja continha 11 fileiras de seis plantas representantes dos clones de batata-doce e uma fileira com o tomate cv. Santa Clara.

O experimento foi instalado no dia 25/01/2013 e para o plantio, utilizaram-se ramas de batata-doce com três a quatro gemas internodais e $20 \mathrm{~cm}$ de comprimento. Em 09/02/2013, 14 dias após o plantio das ramas de batata-doce, realizou-se o semeio na extremidade de cada bandeja da cv. Santa Clara.

Para proceder à infestação do substrato, previamente foi efetuada a extração dos ovos a partir de plantas de tomateiro suscetíveis mantidos em casa de vegetação e altamente infectados por populações de $M$. javanica. Para a obtenção do inóculo, utilizou-se a metodologia descrita por Hussey \& Barker (1973). Para quantificação, os ovos coletados na peneira de menor abertura foram transferidos, com auxílio de uma piceta com água para um béquer, e seu volume ajustado para um litro. Três alíquotas de $1 \mathrm{~mL}$ de suspensão foram transferidas para câmera de Peters e levadas ao estereomicroscópio onde determinou-se o número médio de ovos por $\mathrm{mL}$, procedendo em seguida, a calibração da suspensão na concentração desejada.

As plantas foram inoculadas em 25/02/2013, depositando-se 2.400 ovos de $M$. javanica por célula ao redor do colo das plantas com auxílio de seringa automática de uso veterinário. Em câmara de eclosão foi avaliada a viabilidade do inóculo, e obtiveram-se $57,32 \%$ de ovos viáveis, o que corresponde a 1.375 ovos viáveis de $M$. javanica aplicados por planta.

Em 10/04/2013, decorridos 45 dias após a infestação do substrato, foi realizada a avaliação das plantas. Procedeu-se à cuidadosa lavagem do sistema radicular em água corrente, para a retirada completa do substrato de cultivo das raízes de batata-doce e do tomateiro, evitando-se o jato d'água direto em suas raízes, seguido da extração dos ovos de nematoides, conforme a metodologia proposta por Hussey \& Barker (1973). Logo após, foi quantificado o número total de ovos através da extrapolação da contagem de $1 \mathrm{~mL}$ da suspensão, em câmara de Peters por estereomicroscópio. A partir deste procedimento obteve-se a população final $\left(\mathrm{P}_{\mathrm{f}}\right)$ dos nematoides nas plantas de batata-doce e tomate.

As seguintes variáveis foram utilizadas para verificar a resistência dos clones de batata-doce a M. javanica: Índice de reprodução e Fator de reprodução. $\mathrm{O}$ fator de reprodução foi determinado pela fórmula:

$$
F R=\frac{P_{f}}{P_{i}}
$$

Onde: $F R=$ Fator de reprodução, $P_{f}$ 
$=$ População final e $\mathrm{P}_{\mathrm{i}}=$ População final de ovos viáveis. Segundo Oostenbrink (1966), os clones com FR igual ou maior que 1,0 são consideradas suscetíveis (S) e com valor menor que 1,0 , resistentes (R).

O calculo do índice de reprodução (IR) foi realizado considerando o tomateiro como padrão de suscetibilidade $(100 \%)$ em relação à reprodução dos nematoides obtida nos clones de batata-doce. Assim, os valores obtidos da população final $\left(\mathrm{P}_{\mathrm{f}}\right)$ da batata-doce, foram divididos pela população final encontrados no tomateiro cv. Santa Clara. Os valores resultantes do cálculo para o índice de reprodução permitem a classificação dos clones de batata-doce, de acordo com a classificação estabelecida por Taylor (1967), onde: S, planta suscetível, reprodução normal, IR acima de $51 \%$; LR, levemente resistente, IR de 26 a $50 \%$; MoR, moderadamente resistente, com IR de 11 a $25 \%$; MR, muito resistente, IR de 1 a $10 \%$; AR/I, altamente resistente/imune, IR abaixo de $1 \%$.

Para atender as pressuposições da análise estatística, os valores obtidos pelas classificações de resistência utilizadas, obtidos por contagem, foram submetidos à transformação de $\log (\mathrm{x}+2)$, porém, nas tabelas são apresentados os valores originais. Os dados foram submetidos à análise de variância, e quando identificadas diferenças significativas pelo teste $F$, foram agrupadas pelo teste de Scott-Knott a 5\% de probabilidade. Foi estimada também a herdabilidade no sentido amplo $\left(h_{a}^{2}=\frac{v_{g}}{v_{\varepsilon}}\right)$, o coeficiente de variação genético $C V_{g}(\%)=\frac{\sqrt{V_{g}}}{m} \times 100$, coeficiente de variação ambiental $C V_{e}(\%)=\frac{\sqrt{V_{\varepsilon}}}{m} x 100$, o coeficiente de variação relativo $\left(\mathrm{CV}_{\mathrm{r}}\right)$, representado pela relação entre o coeficiente de variação genético e ambiental $\left(\mathrm{CV}_{\mathrm{g}} / \mathrm{CV}_{\mathrm{e}}\right)$. Para a análise estatística, utilizou-se o software genético-estatístico Genes (Cruz, 2013).

\section{RESULTADOS E DISCUSSÃO}

A partir da análise de variância verificou-se a presença de efeitos

Tabela 1. Códigos antigos e atuais dos clones de batata-doce do banco de germoplasma da UFVJM, após nova padronização nominal (old and current codes of sweet potato clones from the UFVJM germplasm bank, after new nominal standardization). Diamantina, UFVJM, 2013.

\begin{tabular}{|c|c|c|c|}
\hline Código antigo & Código atual & Código antigo & Código atual \\
\hline Arruba & Arruba & BD-44 & UFVJM-27 \\
\hline Batata mandioca & Batata mandioca & BD-46 & UFVJM-28 \\
\hline Brazlândia Branca & Brazlândia Branca & BD-47 & UFVJM-30 \\
\hline Brazlândia Rosada & Brazlândia Rosada & BD-48 & UFVJM-31 \\
\hline Cambraia & Cambraia & BD-50 & UFVJM-32 \\
\hline Cariru vermelha & Cariru vermelha & BD-52 & UFVJM-33 \\
\hline Coquinho & Coquinho & BD-53 & UFVJM-34 \\
\hline Espanhola & Espanhola & BD-54 & UFVJM-35 \\
\hline Licuri & Licuri & BD-55 & UFVJM-36 \\
\hline Palmas & Palmas & BD-56 & UFVJM-37 \\
\hline Princesa & Princesa & BD-57 & UFVJM-38 \\
\hline T Carro 1 & T Carro 1 & BD-62 & UFVJM-39 \\
\hline T Carro 2 & T Carro 2 & BD-65 & UFVJM-40 \\
\hline BD-02 & UFVJM-01 & BD-70 & UFVJM-43 \\
\hline BD-03 & UFVJM-02 & BD-111 TO & UFVJM-44 \\
\hline BD-04 & UFVJM-03 & BD-113 TO & UFVJM-45 \\
\hline BD-05 & UFVJM-04 & BD-31 TO & UFVJM-46 \\
\hline BD-06 & UFVJM-05 & $106-63$ & UFVJM-47 \\
\hline BD-07 & UFVJM-06 & BDI-155 & UFVJM-48 \\
\hline BD-12 & UFVJM-08 & $106-20$ & UFVJM-49 \\
\hline BD-13 & UFVJM-09 & $106-43$ & UFVJM-50 \\
\hline BD-17 & UFVJM-10 & PA-36 & UFVJM-51 \\
\hline BD-23 & UFVJM-13 & PA-26 & UFVJM-52 \\
\hline BD-24 & UFVJM-14 & $02-39$ & UFVJM-53 \\
\hline BD-27 & UFVJM-17 & BDI-158 & UFVJM-55 \\
\hline BD-33 & UFVJM-19 & PA-40 & UFVJM-56 \\
\hline BD-35 & UFVJM-21 & PA-18 & UFVJM-57 \\
\hline BD-36 & UFVJM-22 & $106-06$ & UFVJM-59 \\
\hline BD-38 & UFVJM-23 & 04-05 & UFVJM-60 \\
\hline BD-39 & UFVJM-24 & Sta. Rita do Araguaia & UFVJM-61 \\
\hline BD-42 & UFVJM-25 & BD-18 & UFVJM-63 \\
\hline BD-43 & UFVJM-26 & - & - \\
\hline
\end{tabular}

significativos nos clones para ambas variáveis em estudo, pelo teste $F$. Os coeficientes de variação ambiental para o fator de reprodução e índice de reprodução foram, respectivamente de 19,79 e 8,39, indicando precisões de média a alta no controle ambiental. As estimativas do coeficiente de variação genética apresentaram valores de 58,81 para o fator de reprodução e 10,56 para o índice reprodução; estas estimativas apresentam-se superiores ao coeficiente de variação ambiental, indicando que a variação genética obteve maior expressão em relação ao efeito do ambiente.

Os valores de $96,36 \%$ e $82,62 \%$ das estimativas de herdabilidade no sentido amplo obtidos para o fator de reprodução e índice de reprodução, respectivamente, foram altos, reforçando o fato de que a maior parte da variabilidade fenotípica foi devida a efeitos genéticos. 
Tabela 2. Fatores de reprodução (FR) e índices de reprodução (IR\%) de Meloidogyne javanica em 63 clones de batata-doce e na cultivar de tomate Santa Clara, e classificação desses clones quanto a resistência ou suscetibilidade aos nematoides \{reproduction factor (FR) and reproduction index (IR\%) for Meloidogyne javanica on 63 sweet potato clones and the Santa Clara tomato cultivar, and the rating of these clones for resistance or susceptibility to Meloidogyne javanica\}. Diamantina, UFVJM, 2013.

\begin{tabular}{|c|c|c|c|c|}
\hline \multirow{2}{*}{ Clones } & \multicolumn{2}{|c|}{ Fator de reprodução } & \multicolumn{2}{|c|}{ Índice de reprodução } \\
\hline & $\mathbf{F R}^{* *}$ & Classe & IR ** & Classe \\
\hline Arruba & $0,06 a^{1}$ & $\mathrm{R}^{2}$ & $0,37 \mathrm{a}$ & $\mathrm{AR} / \mathrm{I}^{3}$ \\
\hline Batata mandioca & $6,95 \mathrm{~d}$ & S & $25,87 \mathrm{a}$ & MoR \\
\hline Brazlândia Branca & $0,02 \mathrm{a}$ & $\mathrm{R}$ & $0,04 \mathrm{a}$ & $\mathrm{AR} / \mathrm{I}$ \\
\hline Brazlândia Rosada & $0,11 \mathrm{a}$ & $\mathrm{R}$ & $0,66 \mathrm{a}$ & $\mathrm{AR} / \mathrm{I}$ \\
\hline Cambraia & $4,01 \mathrm{c}$ & $\mathrm{S}$ & $15,08 \mathrm{a}$ & MoR \\
\hline Cariru vermelha & $4,17 \mathrm{c}$ & $\mathrm{S}$ & $13,82 \mathrm{a}$ & MoR \\
\hline Coquinho & $0,23 \mathrm{a}$ & $\mathrm{R}$ & $0,43 \mathrm{a}$ & $\mathrm{AR} / \mathrm{I}$ \\
\hline Espanhola & $2,91 \mathrm{c}$ & $\mathrm{S}$ & $11,74 a$ & MoR \\
\hline Licuri & $0,60 \mathrm{a}$ & $\mathrm{R}$ & $2,10 \mathrm{a}$ & MR \\
\hline Palmas & $0,13 \mathrm{a}$ & $\mathrm{R}$ & $0,45 \mathrm{a}$ & $\mathrm{AR} / \mathrm{I}$ \\
\hline Princesa & $3,87 \mathrm{c}$ & $\mathrm{S}$ & $8,98 \mathrm{a}$ & MR \\
\hline T Carro 1 & $5,37 \mathrm{~d}$ & $\mathrm{~S}$ & $17,34 \mathrm{a}$ & MoR \\
\hline T Carro 2 & $0,55 \mathrm{a}$ & $\mathrm{R}$ & $1,36 \mathrm{a}$ & MR \\
\hline UFVJM-01 & $0,00 \mathrm{a}$ & $\mathrm{R}$ & $0,00 \mathrm{a}$ & $\mathrm{AR} / \mathrm{I}$ \\
\hline UFVJM-02 & $0,19 \mathrm{a}$ & $\mathrm{R}$ & $0,54 \mathrm{a}$ & $\mathrm{AR} / \mathrm{I}$ \\
\hline UFVJM-03 & $3,42 \mathrm{c}$ & $\mathrm{S}$ & $11,90 \mathrm{a}$ & MoR \\
\hline UFVJM-04 & $1,36 \mathrm{c}$ & S & $4,72 \mathrm{a}$ & MR \\
\hline UFVJM-05 & $0,05 \mathrm{a}$ & $\mathrm{R}$ & $0,11 \mathrm{a}$ & $\mathrm{AR} / \mathrm{I}$ \\
\hline UFVJM-06 & $0,24 \mathrm{a}$ & $\mathrm{R}$ & $0,60 \mathrm{a}$ & $\mathrm{AR} / \mathrm{I}$ \\
\hline UFVJM-08 & $5,98 \mathrm{~d}$ & $\mathrm{~S}$ & $20,55 \mathrm{a}$ & MoR \\
\hline UFVJM-09 & $0,10 \mathrm{a}$ & $\mathrm{R}$ & $0,11 \mathrm{a}$ & $\mathrm{AR} / \mathrm{I}$ \\
\hline UFVJM-10 & $0,08 \mathrm{a}$ & $\mathrm{R}$ & $0,34 \mathrm{a}$ & $\mathrm{AR} / \mathrm{I}$ \\
\hline UFVJM-13 & $0,10 \mathrm{a}$ & $\mathrm{R}$ & $0,11 \mathrm{a}$ & $\mathrm{AR} / \mathrm{I}$ \\
\hline UFVJM-14 & $0,10 \mathrm{a}$ & $\mathrm{R}$ & $0,11 \mathrm{a}$ & $\mathrm{AR} / \mathrm{I}$ \\
\hline UFVJM-17 & $0,25 \mathrm{a}$ & $\mathrm{R}$ & $0,92 \mathrm{a}$ & $\mathrm{AR} / \mathrm{I}$ \\
\hline UFVJM-19 & $0,00 \mathrm{a}$ & $\mathrm{R}$ & $0,00 \mathrm{a}$ & $\mathrm{AR} / \mathrm{I}$ \\
\hline UFVJM-21 & $4,71 \mathrm{c}$ & $\mathrm{S}$ & $12,88 \mathrm{a}$ & MoR \\
\hline UFVJM-22 & $39,43 f$ & $\mathrm{~S}$ & $139,99 \mathrm{c}$ & $\mathrm{S}$ \\
\hline UFVJM-23 & $0,15 \mathrm{a}$ & $\mathrm{R}$ & $0,88 \mathrm{a}$ & $\mathrm{AR} / \mathrm{I}$ \\
\hline UFVJM-24 & $1,24 b$ & $\mathrm{~S}$ & $3,56 a$ & MR \\
\hline UFVJM-25 & $0,05 \mathrm{a}$ & $\mathrm{R}$ & $0,26 a$ & $\mathrm{AR} / \mathrm{I}$ \\
\hline UFVJM-26 & $0,13 \mathrm{a}$ & $\mathrm{R}$ & $0,67 \mathrm{a}$ & $\mathrm{AR} / \mathrm{I}$ \\
\hline UFVJM-27 & $0,56 \mathrm{a}$ & $\mathrm{R}$ & $1,68 \mathrm{a}$ & MR \\
\hline UFVJM-28 & $0,06 \mathrm{a}$ & $\mathrm{R}$ & $0,37 \mathrm{a}$ & $\mathrm{AR} / \mathrm{I}$ \\
\hline UFVJM-30 & $0,19 a$ & $\mathrm{R}$ & $0,22 \mathrm{a}$ & $\mathrm{AR} / \mathrm{I}$ \\
\hline UFVJM-31 & $0,05 \mathrm{a}$ & $\mathrm{R}$ & $0,29 \mathrm{a}$ & $\mathrm{AR} / \mathrm{I}$ \\
\hline UFVJM-32 & $0,32 \mathrm{a}$ & $\mathrm{R}$ & $0,97 \mathrm{a}$ & $\mathrm{AR} / \mathrm{I}$ \\
\hline UFVJM-33 & $0,46 a$ & $\mathrm{R}$ & $1,78 \mathrm{a}$ & MR \\
\hline UFVJM-34 & $14,90 \mathrm{e}$ & $\mathrm{S}$ & $54,88 \mathrm{~b}$ & $\mathrm{~S}$ \\
\hline
\end{tabular}

Continua ...
Valores semelhantes de herdabilidade também foram descritos por Marchese $e t$ al. (2010) e Kalkmann et al. (2013), corroborando estes dados. Altos valores de herdabilidade condicionarão maior efetividade na seleção de plantas resistentes à $M$. javanica, para as características avaliadas, fato este reforçado pelo coeficiente de variação relativo, que apresentou valores de 2,97 e 1,26 para fator de reprodução e índice de reprodução, respectivamente. Segundo interpretação recomendada por Vencovsky (1978), a seleção de materiais genéticos se torna favorável quando a relação $\left(\mathrm{CV}_{\mathrm{g}} / \mathrm{CV}_{\mathrm{e}}\right)$ se apresenta acima da unidade, fato que foi verificado no presente estudo, indicando situação muito favorável para seleção.

O fator de reprodução apresentou valor 0,00 para sete clones de batata-doce (UFVJM-01, UFVJM-19, UFVJM-39, UFVJM-43, UFVJM-46, UFVJM-47 e UFVJM-59) e 48,65 para o controle (cv. de tomate Santa Clara), comprovando a viabilidade do inóculo de $M$. javanica utilizado (Tabela 2).

Considerando a classificação proposta por Oostenbrink (1966), 71,43\% dos clones de batata-doce avaliados foram considerados resistentes ao $M$. javanica, apresentando fator de reprodução abaixo de 1,0. A classificação dos clones quanto à resistência é reforçada pelo teste de Scott-Knott, uma vez que os clones considerados resistentes pertencem a grupos estatisticamente distintos do tomateiro cv. Santa Clara e também dos clones de batata-doce classificados como suscetíveis, que por sua vez representam apenas $28,57 \%$ dos clones avaliados.

Dentre as cultivares comerciais avaliadas, todas se apresentaram como resistentes ao $M$. javanica de acordo com a classificação de Oostenbrink (1966), exceto a cultivar Princesa, que foi classificada como suscetível. Resultados obtidos por Huang et al. (1986) corroboram os obtidos no presente estudo para as cultivares comerciais (Brazlândia Rosada e Coquinho, mas diferem em relação a cultivar Brazlândia Branca). Das cinco cultivares comerciais presentes neste estudo, quatro (Brazlândia Branca, Brazlândia Rosada, Coquinho e Princesa) são comuns ao experimento realizado por Charchar \& Ritschel 
Tabela 2. Continuação

\begin{tabular}{|c|c|c|c|c|}
\hline \multirow{2}{*}{ Clones } & \multicolumn{2}{|c|}{ Fator de reprodução } & \multicolumn{2}{|c|}{ Índice de reprodução } \\
\hline & $\mathbf{F R} * *$ & Classe & $\mathbf{I R} * *$ & Classe \\
\hline UFVJM-35 & $0,00 \mathrm{a}$ & $\mathrm{R}$ & $0,00 \mathrm{a}$ & $\mathrm{AR} / \mathrm{I}$ \\
\hline UFVJM-36 & $0,23 a$ & $\mathrm{R}$ & $0,58 \mathrm{a}$ & $\mathrm{AR} / \mathrm{I}$ \\
\hline UFVJM-37 & $0,06 \mathrm{a}$ & $\mathrm{R}$ & $0,07 \mathrm{a}$ & $\mathrm{AR} / \mathrm{I}$ \\
\hline UFVJM-38 & $0,15 \mathrm{a}$ & $\mathrm{R}$ & $0,34 \mathrm{a}$ & $\mathrm{AR} / \mathrm{I}$ \\
\hline UFVJM-39 & $0,00 \mathrm{a}$ & $\mathrm{R}$ & $0,00 \mathrm{a}$ & $\mathrm{AR} / \mathrm{I}$ \\
\hline UFVJM-40 & $1,18 b$ & S & $4,20 \mathrm{a}$ & MR \\
\hline UFVJM-43 & $0,00 \mathrm{a}$ & $\mathrm{R}$ & $0,00 \mathrm{a}$ & $\mathrm{AR} / \mathrm{I}$ \\
\hline UFVJM-44 & $0,22 \mathrm{a}$ & $\mathrm{R}$ & $1,34 \mathrm{a}$ & MR \\
\hline UFVJM-45 & $0,24 \mathrm{a}$ & $\mathrm{R}$ & $0,57 \mathrm{a}$ & $\mathrm{AR} / \mathrm{I}$ \\
\hline UFVJM-46 & $0,00 \mathrm{a}$ & $\mathrm{R}$ & $0,00 \mathrm{a}$ & $\mathrm{AR} / \mathrm{I}$ \\
\hline UFVJM-47 & $0,00 \mathrm{a}$ & $\mathrm{R}$ & $0,00 \mathrm{a}$ & $\mathrm{AR} / \mathrm{I}$ \\
\hline UFVJM-48 & $0,15 \mathrm{a}$ & $\mathrm{R}$ & $0,41 \mathrm{a}$ & $\mathrm{AR} / \mathrm{I}$ \\
\hline UFVJM-49 & $0,11 \mathrm{a}$ & $\mathrm{R}$ & $0,17 \mathrm{a}$ & $\mathrm{AR} / \mathrm{I}$ \\
\hline UFVJM-50 & $0,66 \mathrm{a}$ & $\mathrm{R}$ & $3,03 \mathrm{a}$ & MR \\
\hline UFVJM-51 & $0,19 \mathrm{a}$ & $\mathrm{R}$ & $0,22 \mathrm{a}$ & $\mathrm{AR} / \mathrm{I}$ \\
\hline UFVJM-52 & $2,18 \mathrm{~b}$ & S & $6,19 \mathrm{a}$ & MR \\
\hline UFVJM-53 & $9,46 \mathrm{~d}$ & $\mathrm{~S}$ & $30,09 a$ & LR \\
\hline UFVJM-55 & $2,06 b$ & S & $8,02 \mathrm{a}$ & MR \\
\hline UFVJM-56 & $0,03 \mathrm{a}$ & $\mathrm{R}$ & $0,03 \mathrm{a}$ & $\mathrm{AR} / \mathrm{I}$ \\
\hline UFVJM-57 & $0,05 \mathrm{a}$ & $\mathrm{R}$ & $0,29 \mathrm{a}$ & $\mathrm{AR} / \mathrm{I}$ \\
\hline UFVJM-59 & $0,00 \mathrm{a}$ & $\mathrm{R}$ & $0,00 \mathrm{a}$ & $\mathrm{AR} / \mathrm{I}$ \\
\hline UFVJM-60 & $1,37 b$ & S & $3,97 \mathrm{a}$ & MR \\
\hline UFVJM-61 & $0,15 \mathrm{a}$ & $\mathrm{R}$ & $0,34 \mathrm{a}$ & $\mathrm{AR} / \mathrm{I}$ \\
\hline UFVJM-63 & $0,29 a$ & $\mathrm{R}$ & $0,45 \mathrm{a}$ & $\mathrm{AR} / \mathrm{I}$ \\
\hline Santa Clara & $48,65 f$ & $\mathrm{~S}$ & $100 \mathrm{c}$ & $\mathrm{S}$ \\
\hline Média & 2,66 & & 8,07 & \\
\hline
\end{tabular}

${ }^{1}$ Médias seguidas pela mesma letra na coluna não se diferem pelo teste de Scott-Knott a $5 \%$ de probabilidade (means followed by the same letter in the column do not differ by the Scott-Knott test 5\%); Médias expressas com base nos dados não transformados (means based on the unprocessed data); ${ }^{2} \mathrm{R}=$ resistente (resistant); $\mathrm{S}=$ suscetível (susceptible); ${ }^{3} \mathrm{~S}=$ suscetível (reprodução normal), IR acima de 51\% \{susceptible with normal reproduction and IR above 51\% ; $\mathrm{LR}=$ levemente resistente, IR de 26 a 50\% (slightly resistant, IR from 26 to $50 \%$ ); MoR $=$ moderadamente resistente, IR de 11 a 25\% (moderately resistant, IR from 11 to $25 \%$ ); $\mathrm{MR}=$ muito resistente, IR de 1 a $10 \%$ (very resistant plant, IR from 1 to $10 \%$ ); $\mathrm{AR} /$ $\mathrm{I}=$ altamente resistente/imune, IR abaixo de $1 \%$ (highly resistant/immune, IR below 1\%).

(2004). Entretanto, os resultados obtidos no presente estudo contradizem os observados por estes autores, os quais classificaram como resistente somente a cultivar Princesa. Segundo Kalkmann et al. (2013) e Dias-Arieira et al. (2012), algumas discrepâncias entre os resultados obtidos em trabalhos envolvendo resistência a nematoides podem ocorrer, devido diferenças nas metodologias de avaliações de resistência ou em função da variabilidade existente entre os isolados de nematoides utilizados nos experimentos.

De acordo com o índice de reprodução, foi constatada a distribuição dos clones de batata-doce em todas as cinco classes da escala de classificação elaborada por Taylor (1967) (Tabela 2). Entretanto, elevada proporção de clones demonstrou reação altamente resistente/imune ou muito resistente ao $M$. javanica, reação semelhante ao já observado para a classificação de Oostenbrink (1966), onde a maior parte dos clones foi classificada como resistente, demonstrando assim, que os clones no presente estudo podem ser considerados maus hospedeiros para o M. javanica.

Quando se observa a classificação proposta por Taylor (1967) (Tabela 2), os clones classificados como altamente resistente/imune (AR/I) representaram no total $61,90 \%$ dos clones testados, e nestes incluem as cultivares comerciais, Brazlândia Branca, Brazlândia Rosada, Coquinho e Palmas, enquanto a cultivar Princesa encontra-se entre os $20,61 \%$ dos clones classificados como muito resistente (MR). Considerando o mesmo nível de seleção utilizado por Marchese et al. (2010) que definiu como merecedores da seleção somente clones que apresentaram reação de AR/I e MR pelo índice de reprodução, estes clones serão então, aptos a serem selecionados. Em proporções bem menores, $12,69 \%$ e $1,60 \%$ dos clones avaliados foram classificados como moderadamente resistente e levemente resistente, respectivamente.

Quanto à reação dos clones à suscetibilidade, pode-se observar que além do tomateiro cv. Santa Clara, considerado controle suscetível (100\% para o índice de reprodução), outros dois clones, UFVJM-22 e UFVJM-34 apresentam-se suscetíveis para o $M$. javanica, sendo que o clone UFVJM-22 proporcionou aumento de 1,40 vezes no índice de reprodução em relação ao apresentando pelo controle, podendo ser considerado assim, mais suscetível que o controle padrão.

Alta proporção de clones resistentes a Meloidogyne spp. tem sido relatada em experimentos com batata-doce. Charchar \& Ritschel (2004) avaliaram a resistência para 13 populações de espécies e raças de Meloidogyne em 357 clones de batata-doce do banco de germoplasma da Embrapa Hortaliças e verificaram que 222 clones apresentaram reação altamente resistente, sem infecção por nenhuma das populações de nematoides. Maluf et al. (1996) avaliaram a resistência de outros 226 genótipos de batata-doce a $M$. javanica e $M$. 
incognita e obtiveram alta proporção de genótipos resistentes para M. javanica.

Em relação às duas classificações de resistência utilizadas, ambas demonstraram ser úteis na distinção de clones resistentes e suscetíveis para o propósito de seleção de clones resistentes, fato este explicitado nos clones que apresentaram algum grau de resistência e obtiveram médias estatisticamente diferentes $(p<0,05)$ do controle e dos clones considerados suscetíveis. Estas classificações têm sido utilizadas por vários autores em trabalhos similares quanto à seleção para resistência aos nematoides como Melo et al. (2011), Paula et al. (2011), Pilco et al. (2011), Silva et al. (2011) e Dias-Arieira et al. (2012). Entretanto, estas classificações proporcionam informações distintas, pois comparando-as, observa-se que alguns clones que foram classificados como suscetíveis pela classificação de Oostenbrink (1966) apresentaram algum grau de resistência pela classificação de Taylor (1967).

Verifica-se que a classificação pelo fator de reprodução é a mais indicada para utilização no processo de seleção de clones de batata-doce resistentes, uma vez que esta classificação baseia-se somente na relação entre número de ovos dos nematoides final e inicial obtidos na batata-doce. Com isto, os clones que apresentarem $\mathrm{FR}<1$ tendem ao longo do ciclo de cultivo ter a população de nematoides reduzida ou até, a total eliminação dos nematoides, já que o ciclo da cultura é de aproximadamente 120 dias, enquanto, o ciclo de vida dos nematoides do gênero Meloidogyne tem duração aproximada de 30 dias. Sendo então, esta classificação de grande importância para plantas de ciclo longo e propagação assexuada, como é o caso da batata-doce.

Quanto à utilização da classificação pelo índice de reprodução, verificou-se que esta possibilita maior flexibilidade, devido ao ponto de truncagem utilizado para seleção dos clones basear-se em uma distribuição mais ampla de classes (AR/I, MR, MoR, LR e S). No entanto, esta classificação considera os níveis de resistência baseando-se no controle altamente suscetível, o tomate cv. Santa Clara. Neste sentido, para propósitos de seleção de clones resistentes, esta clas- sificação não deve ser a preferível. De forma geral, verifica-se que a utilização do índice de reprodução é mais indicada para fins onde não se deseja realizar uma seleção rigorosa, uma vez que esta classificação baseia-se em várias classes de resistência, enquanto o fator de reprodução se baseia somente em duas classes (resistente ou suscetível).

Devido à dificuldade e ineficiência de se combater Meloidogyne spp. com agroquímicos, o uso de cultivares resistentes é o método que apresenta maior eficiência. Genótipos resistentes a nematoides foram verificados em batata-doce (Kalkmann et al., 2013), genótipos de pimentão (Peixoto et al., 1999), genótipos de alface (Ferreira et al., 2011) e em genótipos de feijão (Santos et al., 2012), todos com base em classificações de resistência. Desta forma, considerando a resistência conjunta por ambas as classificações, as cultivares comerciais (Brazlândia Rosada, Brazlândia Branca, Palmas, Coquinho), e os clones (Arruba, Licuri, T Carro 2, UFVJM-01, UFVJM-02, UFVJM-05, UFVJM-06, UFVJM-09, UFVJM-10, UFVJM-13, UFVJM-14, UFVJM-17, UFVJM-19, UFVJM-23, UFVJM-25, UFVJM-26, UFVJM-27, UFVJM-28, UFVJM-30, UFVJM-31, UFVJM-32, UFVJM-33, UFVJM-35, UFVJM-36, UFVJM-37, UFVJM-38, UFVJM-39, UFVJM-43, UFVJM-44, UFVJM-45, UFVJM-46, UFVJM-47, UFVJM-48, UFVJM-49, UFVJM-50, UFVJM-51, UFVJM-56, UFVJM-57, UFVJM-59, UFVJM-61, UFVJM-63) apresentaram-se resistentes e podem ser utilizados como fontes de resistência a Meloidogyne javanica.

\section{AGRADECIMENTOS}

À Universidade Federal dos Vales do Jequitinhonha e Mucuri (UFVJM); à CAPES, CNPq e FAPEMIG; à Universidade Federal e Lavras (UFLA); à empresa HortiAgro Sementes S.A. e ao Grupo de Estudos em Olericultura/ UFVJM.

\section{REFERÊNCIAS}

CHARCHAR JM; RITSCHEL PS. 2004.
Avaliação do banco de germoplasma de batata-doce da Embrapa Hortaliças para resistência a Meloidogyne spp. Brasília: Embrapa Hortaliças, 28p.

CIP - Centro Internacional de La Papa. 2013, 6 de novembro. Facts and figures about sweet potato. Disponível em: http//:www.cipotato. org.

CRUZ CD. 2013. Genes a software package for analysis in experimental statistics and quantitative genetics. Acta Scientiarum 35: 271-276.

DIAS-ARIEIRA CR；CUNHA TPL; CHIAMOLERA FM; PUERARI HH; BIELA F; SANTANA SM. 2012. Reaction of vegetables and aromatic plants to Meloidogyne javanica and M. incognita. Horticultura Brasileira 30: 322-326.

ECHEVERRIGARAY S; ZACARIA J; BELTRÃO R. 2010. Nematicidal activity of monoterpenoids against the rootknot nematode Meloidogyne incognita. Phytopathology 100: 199-203.

FERREIRA S; VIEIRA VLF; GOMES LAA; MALUF WR; CARVALHO FILHO JLSD. 2011. Identificação de linhagens avançadas de alface quanto à resistência a Meloidogyne javanica. Ciência e Agrotecnologia 35: 270277.

FIORINI CVA; GOMES LAA; LIBÂNIO RA; MALUF WR; CAMPOS VP; LICURSI V; MORETTO P; SOUZA LA; FIORINI IVA. 2007. Identificação de famílias F2:3 de alface homozigotas resistentes aos nematoides das galhas. Horticultura Brasileira 25: 509-513.

HUANG SP; MIRANDA JEC; MALUF WR. 1986. Resistance to root-knot nematodes in Brazilian sweet potato collection. Fitopatologia Brasileira 11: 761-767.

HUSSEY RS; BARKER KR. 1973. A comparison of methods of collecting inocula of Meloidogyne spp. including a new technique. Plant Disease Reporter 57: 1025-1028.

IBGE. Instituto Brasileiro de Geografia e Estatística. 2013. Produção agrícola municipal. Culturas temporárias e permanentes 2011. Disponível em: http://www.ibge.gov.br/home/ estatistica/pesquisas/pesquisa_resultados. php?id_pesquisa $=44$.

JATALA P. 1991. Biology and management of plant-parasitic nematodes on sweet potato In: JANSSON RK; RAMAN KV. Sweet potato pest management: a global overview. Sweet potato pest management: a global perspective. New Delhi: Oxford and IBH publishing. p. 359-378.

KALKMANN DC; PEIXOTO JR; NÓBREGA DDS. 2013. Reaction of sweet potato clones to Meloidogyne incognita races 1 and 4, and estimation of genetic parameters. Horticultura Brasileira 31: 293-296.

MALUF WR; AZEVEDO SM; CAMPOS VP. 1996. Heritability of root knot nematode Meloidogyne spp. resistance in sweet potatoes. Journal of Genetic and Breeding 50: 161-165.

MARCHESE A; MALUF WR; GONÇALVES NETO AC; GONÇALVES RDS; GOMES LAA. 2010. Seleção de clones de batata-doce resistentes a Meloidogyne incognita raça 1. Pesquisa Agropecuária Brasileira 45: $997-$ 
1004.

MARINO RH; GOMES LAA; OLIVEIRA CRUZ EM; SILVA ADC; BIANCHINI FG; MENESES TN; BLANK AF. 2012. Controle de Meloidogyne incognita raça 1 com óleo essencial de Lippia alba. Scientia Plena 8: 1-8.

MELO OD; MALUF WR; GONÇALVES RJS; GONÇALVES NETO AC; GOMES LAA; CARVALHO RC. 2011. Triagem de genótipos de hortaliças para resistência a Meloidogyne enterolobii. Pesquisa Agropecuária Brasileira 46: 829-835

NEVES WDS; DALLEMOLE-GIARETTA R; ZOOCA RJF; COUTINHO MM. 2010. Efeito de extratos botânicos sobre a eclosão e inativação de juvenis de Meloidogyne javanica e de M. incognita. Revista Trópica: Ciências Agrárias e Biológicas 4: 1-8.

OOSTENBRINK M. 1966. Major characteristics of the relation between nematodes and plants. Mededelingen van de landbouwhogeschool te Wageningen 66: 1-46.

PAULA LAD; BIANCHI VJ; GOMES CB; FACHINELLO JC. 2011. Reação de portaenxertos de pessegueiro Meloidogyne incognita. Revista Brasileira de Fruticultura 33: 680-684.

PEIXOTO JR; MALUF WR; CAMPOS VP. 1999. Avaliação de linhagens, híbridos F1 e cultivares de pimentão quanto à resistência a Meloidogyne spp. Pesquisa Agropecuária Brasileira 34: 2259-2265.

PERRY RN; MOENS M; STARR JL. 2009. Rootknot Nematodes. CAB International. 483p.

PILCO M; CROZZOLI GD; PERICHI RYG. 2011 Reacción de selecciones de batata al nematodo agallador Meloidogyne javanica. Fitopatología Venezolana 24: 52-55.

PINHEIRO JB; BOITEUX LS; LOPES CA; SILVA GO. 2009. Identificação de fontes de resistência ao nematoide Meloidogyne mayaguensis em acessos de tomateiro (Solanum Lycopersicon). Brasília: Embrapa Hortaliças, 19p.

SANTOS LNSD; ALVES FR; BELAN LL; CABRAL PDS; MATTA FDP; JUNIOR J; MORAES WBD. 2012. Damage quantification and reaction of bean genotypes (Phaseolus vulgaris L.) to Meloidogyne incognita race 3 and M. javanica. Summa Phytopathologica
38: 24-29.

SILVA GO; PINHEIRO JB; VIEIRA JV; CARVALHO ADF. 2011. Seleção para resistência de genótipos de cenoura aos nematoides-das-galhas. Horticultura Brasileira 29: 335-341.

SILVA JBC; LOPES CA; MAGALHÃES JS. 2008. Embrapa Hortaliças. Sistemas de produção, Versão Eletrônica. Cultura da batata doce. Disponível em: <http://www. cnph.embrapa.br/sistprod/batatadoce/index. htm>. Acessado em: 28 out. 2013.

TAYLOR AL. 1967. Introduction to research on plant nematology. In: FAO guide to study and control of the plant-parasitic nematodes. Rome: Food And Agricultural Organization of the United Nations. 133p.

VENCOVSKY R. 1978. Herança quantitativa. In: PATERNIANI E. Melhoramento de milho no Brasil. Piracicaba: Fundação Cargill. p. 122-199.

WESEMAEL WML; VIAENE N; MOENS M. 2011. Root-knot nematodes (Meloidogyne spp.) in Europe. Nematology, SI 13: 3-16. 